\title{
The origin of pulsating auroras
}

\section{Spectacular light shows in Earth's atmosphere called pulsating auroras are directly linked to processes in space. After decades of research, the full chain of events that creates such auroras has been observed. SEE LETTER P.337}

\section{ALLISON N. JAYNES}

$\mathrm{T}$ he Northern and Southern lights, also known as auroras, are as varied as the colours they display in the night sky. Discrete auroras are the kind that typically grace our desktops and calendar covers, and that are produced a few thousand kilometres above Earth's surface. By contrast, pulsating auroras that are created tens of thousands of kilometres away, in the equatorial region of the magnetosphere - the area around Earth that is dominated by the planet's magnetic field. For decades, it has been suggested that pulsating auroras are the result of interactions between magnetospheric electrons and electromagnetic waves called chorus waves that send electrons careering towards Earth's atmosphere along magnetic-field lines ${ }^{1-3}$. On page 337 , Kasahara et al. ${ }^{4}$ report direct evidence for this process using observations both from Earth's surface and from a spacecraft positioned on a field line.

Because magnetic fields are invisible to the human eye, the prediction of where a field line hits Earth and where that same field line exists out in space - a task known as magneticfield-line mapping - is extremely difficult ${ }^{5}$. Luckily, electrons that move around Earth tend to follow these field lines closely. When these particles interact with chorus waves, they can be directed into a region of the upper atmosphere called the ionosphere, where they often generate auroral light. This allows us instantly to see the footprint of the associated field lines.

In addition, if we have an observation platform at a precise location out in space, we can detect the chorus waves that caused the electrons to head towards the atmosphere and see fluctuations in the electron population that arise from the oscillation of the waves. The trick is to get the ground-based and spacebased observations to line up at the right time and place, and to have instruments sensitive enough to view both processes simultaneously. This feat has eluded observers ever since the theory of pulsating-aurora generation was developed $^{6,7}$.

The first challenge is to have an instrument capable of making the in situ measurement of electrons in space at the required resolution. The Arase spacecraft ${ }^{8}$, launched by the Japan Aerospace Exploration Agency in late 2016,

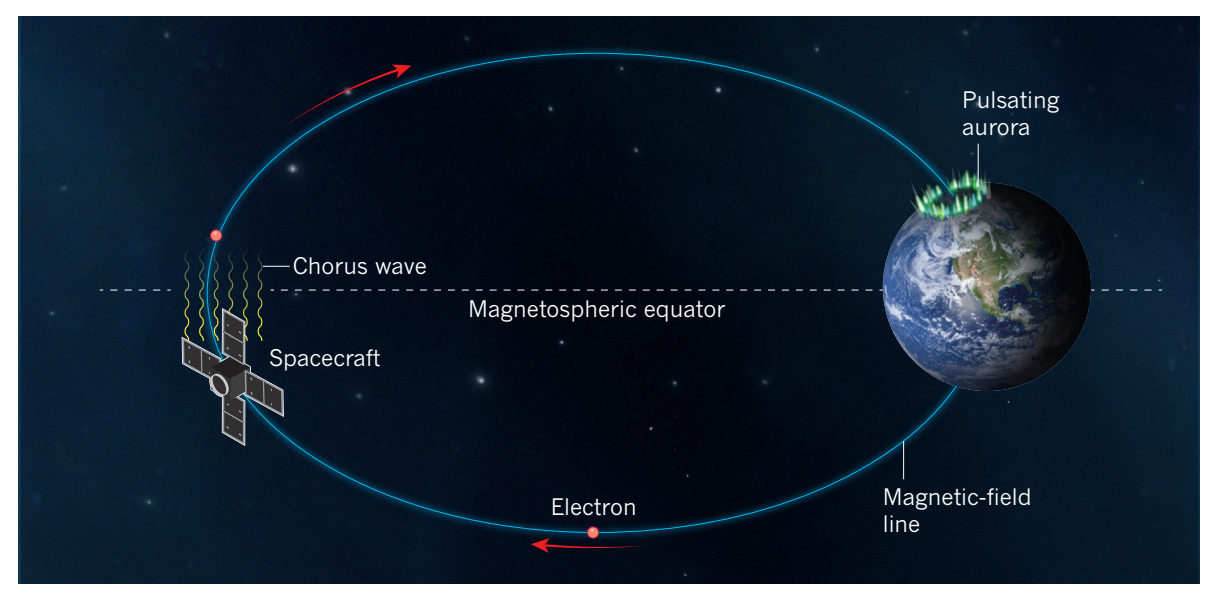

Figure 1 | Pulsating-aurora generation. Kasahara et al. ${ }^{4}$ report evidence for a mechanism that explains the occurrence of blinking patches of light in Earth's atmosphere called pulsating auroras. In a region surrounding Earth known as the magnetosphere, electrons are trapped by the planet's magnetic field and travel (red arrows) along magnetic-field lines. When these particles interact with electromagnetic waves called chorus waves, which are generated in the equatorial region of the magnetosphere, they can be directed towards Earth's atmosphere, where they produce auroral light. The authors detected the interactions between the chorus waves and the electrons using the Arase spacecraft ${ }^{8}$, which was positioned on the relevant field line.

carries just such an electron detector, which was designed to observe electrons within a narrow window around a magnetic-field line. The spacecraft is also equipped with instruments to detect chorus waves. Kasahara and colleagues analysed data from the spacecraft to uncover fluctuations in the electron population and the associated chorus waves.

The next obstacle was to determine where the field line threading the position of the spacecraft would hit the ground. Magnetic-field models are now sophisticated enough to be able to inform researchers of the approximate location of a field-line footprint in Earth's atmosphere, generally with higher accuracy when the level of geomagnetic activity (magnetic storms) is low. In the vicinity of this footprint, Kasahara et al. looked for a corresponding pulsatingaurora signal - namely, variations in aurorallight intensity that matched the fluctuations in the electron population. They identified such a signal in measurements from an all-sky imager based in central Canada ${ }^{9}$, which essentially records black-and-white video of the hemispherical view of the sky above (see Figure 2 of the paper ${ }^{4}$ ).

Thanks to Kasahara and colleagues, we can see the complete process of pulsating-aurora generation for the first time: the fluctuations in an electron population out in space; the chorus waves responsible for these fluctuations; and the variations in auroral-light intensity from the ground (Fig. 1). The last part is somewhat analogous to watching an image on an oldfashioned television, where the ionosphere is the 'screen' onto which electrons are projected. Despite this simple picture, researchers are aware that the ionosphere probably changes the incoming signal - a detail that will no doubt be scrutinized in future studies.

Kasahara et al. carried out an analysis in which they correlated the electron fluctuations and chorus waves in space with the pulsatingaurora signals seen by the all-sky imager on the ground. This step revealed the precise location in the atmosphere in which the field-line footprint resides. Such a technique has incredible potential to test and refine our current magnetic-field models by comparing the modelled footprint location to the observed location. In the future, magnetic-field-line mapping might well rely on a similar methodology to gain insight into magnetic topology - the structure and linkage of field lines. 
There is one caveat, however: clear skies are required to see and measure the pulsatingaurora signals, so Earth's terrestrial weather needs to cooperate. Furthermore, the chorus waves contain components of different frequency that interact with magnetospheric electrons in different ways depending on the energy of the particles. This affects which particles end up travelling down to Earth's atmosphere. These details are directly related to geomagnetic activity and have not yet been fully quantified. There is still a rich body of research to be carried out regarding the mysterious pulsating auroras.

Allison N. Jaynes is in the Department of Physics and Astronomy, University of Iowa, Iowa City, Iowa 52242, USA.

e-mail:allison-n-jaynes@uiowa.edu

1. McEwen, D. J., Yee, E., Whalen, B. A. \& Yau, A. W Can. J. Phys. 59, 1106-1115 (1981).

2. Nishimura, Y. et al. J. Geophys. Res. Space Phys.
116, A11221 (2011).

3. Jaynes, A. N. et al. J. Geophys. Res. Space Phys. 118, 4884-4894 (2013)

4. Kasahara, S. et al. Nature 554, 337-340 (2018).

5. Pulkkinen, T. I. \& Tsyganenko, N. A. J. Geophys. Res. 101, 27431-27442 (1996)

6. Coroniti, F. V. \& Kennel, C. F. J. Geophys. Res. 75, 1279-1289 (1970)

7. Davidson, G. T. J. Geophys. Res. 84, 6517-6523 (1979).

8. Miyoshi, Y. et al. J. Phys. Conf. Ser. 869, 012095 (2017).

9. Mende, S. et al. Space Sci. Rev. 141, 357-387 (2008).

it undermines reliable testing of a hypothesis ${ }^{9}$.

Ashton and colleagues turned instead to intraspecies comparisons. They studied 56 magpies, which were ringed to enable identification, from 14 different territorial groups that ranged in size from 3 to 12 birds (Fig. 1). Rather than measuring brain size, the authors conducted cognitive-performance tests in which the birds encountered wooden or plastic devices that tested problem-solving skills; successful birds received a mozzarellacheese treat. Four different devices each tested a specific skill, including spatial memory and the ability to learn new associations between stimuli and rewards.

The authors report that group size is linked to cognitive performance. Birds living in a larger group displayed better performance at the population level on each of the tests than did birds living in smaller groups. At the individual level, exceptions to this trend could be found - some birds from smaller groups outperformed birds from larger groups, and vice versa.

The authors recorded the reproductive success of individuals by counting the average number of hatched clutches of eggs per year, and found that birds that performed well on the tests had greater reproductive success than such animals live in a group become the main selective force driving the evolution of primate intelligence. On page 364, Ashton et al. ${ }^{2}$ offer support for Humphrey's social-intelligence hypothesis, in a study of wild Australian magpies (Cracticus tibicen dorsalis, also known as Gymnorhina tibicen dorsalis).

The causal relationships between social complexity, intelligence and reproductive success proposed by Humphrey inspired a generation of primatologists, who uncovered unexpected sophistication in monkeys' and apes' social knowledge and political manoeuvrings $s^{3-5}$. However, these discoveries arguably made it difficult to test Humphrey's hypothesis directly, because intelligence both in social interactions and in non-social realms, such as foraging or tool use - and social complexity were revealed to be composed of many components ${ }^{4,5}$. Primate social complexity, like intelligence itself, was found to be extraordinarily complex.

In 1995, primatologist Robin Dunbar suggested ${ }^{6}$ that focusing on the typical group size of a species as a proxy for social complexity, and on its brain size instead of intelligence,

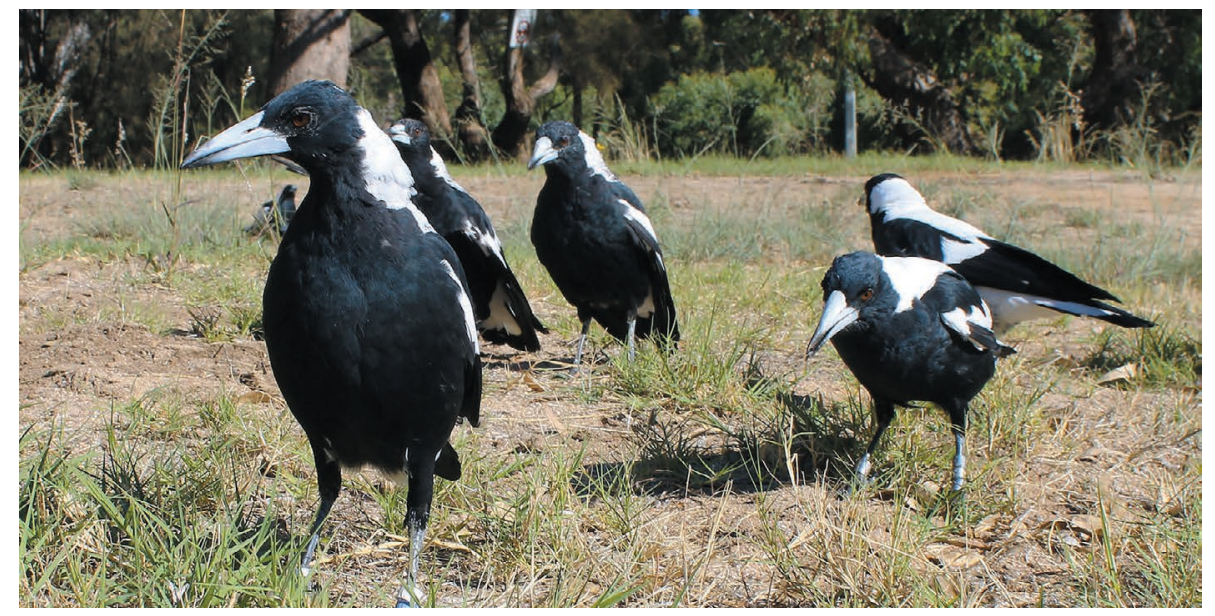

Figure 1 | Australian magpies in Guildford, Western Australia. Wild Australian magpies (Cracticus tibicen dorsalis, also known as Gymnorhina tibicen dorsalis) are territorial and live in groups. Ashton et al. ${ }^{2}$ analysed the relationship between the birds' group size and cognitive performance to test the long-debated idea ${ }^{1}$ that life in complex social groups can select for intelligence. At the population level, larger groups of birds performed better than smaller groups in cognitive-performance tests, and cognitive performance was also linked to reproductive success. 